\title{
Mixed Reality Benefits For Design Perception
}

\author{
by \\ Phillip S. Dunston ${ }^{1}$, Xiangyu Wang ${ }^{2}$, Mark Billinghurst ${ }^{3}$, Ben Hampson ${ }^{4}$ \\ ${ }^{I}$ Construction Engineering and Management Division \\ School of Civil Engineering \\ Purdue University \\ West Lafayette, Indiana \\ (dunston@ecn.purdue.edu) \\ ${ }^{2}$ Department of Civil and Environmental Engineering \\ University of Washington \\ Seattle, Washington \\ ${ }^{3}$ Human Interface Technology Laboratory \\ University of Washington \\ Seattle, Washington \\ ${ }^{4}$ The McKinstry Company, Seattle, Washington
}

\begin{abstract}
Design visualization is key to the communication and shared perception of designs and is essential for meaningful design development and collaborations. The initial development of an Augmented Reality Computer Aided Drawing (AR CAD) system for enhancing visualization of models created in standard CAD was presented at the 17th ISARC. AR CAD features a more natural mode for changing views of the model and completely understanding the model content. Expected benefits are improved efficiency in the design detailing function, both for the individual detailer and for design collaborations where maintaining an accurate shared understanding of the design model is critical. An experimental program is under way to examine the impact of AR CAD upon a user's perception and recall of a design model. Related experiments with desktop and immersive virtual environments have found that motion cues can indeed markedly improve spatial cognition. It is expected that we will see the same benefits in our AR CAD system, although until now such studies have not been conducted in an AR environment. This paper presents the rationale for experiments to measure the impact of $\mathrm{AR} C \mathrm{CAD}$ in terms of cognition cost, and it lays the foundation for further application of Mixed Reality (MR) technology to the design, construction, and maintenance phases of a facility's life cycle. MR applications may prove promising for effective communication of designs for prefabrication, site installation, and the planning and excecution of maintenance operations.
\end{abstract}

KEYWORDS: 3D CAD; Augmented Reality; Mixed Reality; spatial cognition; visualization; Virtual Reality

\section{INTRODUCTION}

Recognizing 3D CAD and walk-through software as the state-of-the-art for visualizing design details, Augmented Reality Computer Aided Drawing (AR CAD) was introduced at the 17 th ISARC [1]. In addition to providing an alternative three-dimensional view of construction models, it was proposed that experimentation with the spectrum of Mixed Reality as illustrated by Milgram and Kishino [2] in Figure 1 could open the door to more modes of interaction with design content than is currently available through typical CAD software. Alternative interface metaphors can be developed and tailored to facilitate development and communication of designs. Thus, our work is aimed at determining the 
appropriate MR modes for planning, design, construction, maintenance and the associated interface tools.

Augmented Reality (AR) occupies that place in the continuum where virtual objects are inserted to a predominantly real world scene. AR also allows virtual enhancements to physical interface objects. MR offers a broader range of options for interfacing with digitally based information. In our present work we are interested in applying more innovative AR techniques to the design phase of the construction process, the AR CAD system is designed for exploring the benefits of supporting design, and ultimately construction, with various modes of Virtual Reality interfaces.

The AR CAD system utilizes fiduciary markers in the real world to position the model and allow the user to easily see it from any viewpoint. As such, it offers the benefit of a very natural mode for changing views of the model and completely understanding the content than would be afforded by visualization systems that have a more constrained means of navigation. This feature is expected to improve efficiency in the design detailing function, both for the individual detailer and for design collaborations where maintaining an accurate shared understanding of the design model is critical.

An experimental program is underway to examine the impact of AR CAD upon a user's perception and recall of a design model. Related experiments with desktop and immersive virtual environments have found that motion cues can indeed markedly improve spatial cognition. It is expected that we will see the same benefits in AR CAD, although until now such studies have not been conducted in an AR environment. Positive results from these experiments would confirm that AR CAD has the potential to support the reduction of errors during design detailing and the more rapid and effective resolution of space conflicts interferences during design collaborations. This stage of research lays the groundwork for further application of MR technology to the design, construction, and maintenance phases of a facility's life cycle. MR applications may prove promising for effective communication of designs for prefabrication, site installation, and the planning and execution of maintenance operations.

This paper presents the updated description of the features of the AR CAD system and also explores some spatial cogniton issues that may arise associated with the system. We also explain the rationale for experiments to determine the benefits of AR CAD over standard CAD (AutoCAD in this instance) in conflict detection. Such experiments constitute our first attempt to evaluate AR CAD with regard to spatial cognition issues.

\section{AR CAD SYSTEM}

The AR CAD system has been modified for improved performance over its predecessor version. The current experimental facility can provide the piping detailer with the ability to explore the CAD design in the non-immersive (AR) virtual reality mode and still consists of the following components as first described by Dunston et al. [1] and shown in Figure 2.

Modeling Computer: The modeling computer runs AutoCAD and a specially designed AutoCAD plug-in. The CAD detailer designs the model on this machine and then sends the 3D model information out to the AR computer using the AutoCAD plug-in. The plug-in software uses standard network communication code to communicate between computers. Communication can also be between modules in the same computer. The current system functions on a single desktop Pentium 4 PC with $1.6 \mathrm{GHz}$.

AR Computer: The AR computer runs an Augmented Reality application that allows a user to see virtual 3D models superimposed over a real-time video-recorded view of the real world. The AR application is a custom application that is based on the ARToolKit library [3] and OpenGL library [4] and a database, containing simplified 3D models of pipe structures in the VRML file format. The AR application receives the 3D model information through the network communication and then instantly creates a $3 \mathrm{D}$ virtual model of the design. 
Camera: Also connected to the AR computer is a Logitech QuickCam Pro 3000 Camera with video capture of up to $640 * 480$ pixels and frame rate of up to 30 frames per second. The computer performs image processing on the video image from the camera to find specially marked tracking cards. The camera's position can be calculated from a tracking card and a virtual model overlaid on the card. The resultant composite image is fed back into the desktop display for the user to see. The result is a view of the real world scene with a 3D virtual model overlaid on it (Figure 3). This tracking technique enables the user to easily view the model from any perspective above the card.

\section{NEW FEATURES IN THE SYSTEM}

Several feature modifications or additions have been made to the AR CAD prototype. These features are as follows:

1. New Graphics Library: The previous version of $A R$ CAD relied upon the LibVRML97 library for the rendering of virtual images while the newer version relies upon the OpenGL standard for greater versatility and generation of more stable virtual models. OpenGL is a graphics library that is less memory intensive and facilitates real-time interactions with the virtual models.

2. Automatic Conflict Detection: The program will automatically detect any conflict or interference appearing among the pipe objects. If there is a conflict, the interfered objects will be highlighted as wire frame elements on the screen rather than the default solid model representation (see Figure 4). This task is accomplished using the boundary box feature of OpenGL. A similar conflict detection function has been developed by Shiau et al.[5] whose application uses ellipses to identify interferences in structures, appliances and piping systems in 3D models.

3.Objects Selection and Manipulation: If a certain object is selected (activated), the wire frame of it will appear on the scene, which makes the designer easily recognize which object is activated so that the user can use the mouse to move and scale any object activated and also use the keyboard to rotate the object along local $\mathrm{x}, \mathrm{y}, \mathrm{z}$ axes. Brief information describing the selected object will be shown as a text string in the bottom of the screen.

A zooming feature has also been added to the AR module (see Figure. 5). Another potentially beneficial function under development is a transparency mode. This rendering mode can provide distant objects with a degree of visibility even if the view is obstructed by nearer objects. Finally, we are in the midst of adding a fly-in feature that will provide an immersive VR view of the design space.

\section{SPATIAL COGNITION COST}

Since AR CAD presently acts mostly as an assistant viewing tool for standard AutoCAD, the chief issues that have arisen are those concerning human spatial cognition. While 3D modeling is readily accepted as being less abstract and therefore an intrisically more meaningful graphic form for representing and communicating complex designs, there are still open questions due to the degree of separation from reality that yet remains, questions relating to control of perspective and user interface metaphors. Some features or options of the AR CAD software raise the question of costs and benefits of AR CAD with respect to spatial cognition. That is, do the spatial cognition benefits outweigh the cognition cost associated with a viewing assistant mode?

Very limited research has been done on spatial cognition issues associated with AR applications. However, some testing results done by other researchers indicate that subjects are able to acquire configuration knowledge of immersive virtual environments in spite of the fact that the subjects lack the benefit of spatial calibration derived from physical movements through a real environment. In this paper, we are concerned with the time cost of necessary cognitive processes. 
During construction of spatial mental models, switching perspective from one scene to another scene exacts a cost [6]. Cognition cost is a kind of cognitive cost incurred by mental transformations associated with the such changes as a new referent object or frame, or change of a viewpoint while switching perspectives. Perspective switching due to transitions between AutoCAD and the AR scene will incur a cognition cost (see Figure 6 for comparison of AR CAD model and AutoCAD model). However, presently, we don't know how much spatial cognition benefit - a more natural and more smooth navigation between viewpoints - AR CAD can provide versus the cognition cost of using the system as we have designed it to presently operate.

To understand what that cost may be, we need to analyze what perspective switching entails. A perspective consists of a referent object or frame and a viewpoint. Each of these components changes when perspective is changed. Each of these changes requires different mental transformations and cognition cost associated with them. However, the relative costs of the transformation are not yet fully known.

The method for changing viewpoint in the AR scene is quite different from that used in AutoCAD. For AR scene navigation, the viewpoint is embedded in real world background and keeps changing as the user changes the relative position between the camera and the tracking marker. In contrast, standard CAD software like AutoCAD has a single color background with only a simple symbol referencing the coordinate system origin. There is nothing else in the scene to which the user can reference his or her viewing perspective. Furthermore, the mechanisms for changing views is often not intuitive.

Even though the relative position of each object in the AR scene is absolutely the same as the one in AutoCAD, another significant cognition cost will come from additional mental processing demands for reconciling misaligned headings. When transitioning between scenes (AR and AutoCAD) in which models are misaligned, mental calculations are required to account for the different headings in each scene. This condition is very similar to alignment effects found for map usage [7]. If an additional step is necessary to compute the direction of a location for misaligned headings, there is a resulting processing demand and cognition cost.

While there is indeed a cognition cost associated with switching scenes or perspectives, in some cases, the costs of switching may not be greater than the cost of staying with the same scene. One of the major advantages of AR CAD is a natural and smooth transition between viewpoints making it easier to locate any point or corner of the virtual scene quickly. However, the big drawback internal to $\mathrm{AR} \mathrm{CAD}$ is the cognition cost in transitioning between the virtual scene in AR and the original model in AutoCAD. There is a trade-off in utilizing these two viewing environments together. However, right now, we do not know how much spatial cognition benefit is associated with AR $\mathrm{CAD}$, nor how much cognition cost. If a specific benefit is identified, then the cognition cost for obtaining it can be measured for standard CAD (AutoCAD) alone and for CAD equipped with the AR viewing mode. This approach can be used to validate any sort of viewing assist mode or function. For instance, conflicts or interferences can be identified in the AutoCAD view by means of certain visible features or by transitioning to the AR scene to use the automatic conflict detection feature and then returning to AutoCAD to make corrections. The simple measurement of time indicates the relative cognition cost. We are performing such tests, incorporating statistical design to allow for differences between user subjects and model complexity. Preliminary trials have indicated the AR viewer assist to be a worthwhile means for identifying conflicts without increasing a detailer's overall time in detecting conflicts.

\section{CONCLUSION}

This paper has presented an update on the development of an Augmented Reality viewer assist feature that turns standard CAD into AR $\mathrm{CAD}$ and has discussed the importance of considering spatial cognition issues in the validation of this system. Future work will pursue 
confirmation of AR CAD benefits for design model perception for both individual users and collaborating partners. These efforts will also include feature enhancements to extend AR CAD's applicability, such as more seamless generation of the virtual models. The long term objective is the validation of Mixed Reality as a useful technology arena for effecting human interfaces with digitally based project design information.

\section{ACKNOWLEDGEMENTS}

The authors are grateful to the McKinstry Company of Seattle, Washington (U.S.A.) for their continuing cooperation in this research.

\section{REFERENCES}

1. Dunston, P.S., Billinghurst, M., Luo, Y. and Hampson, B. 2000. "Virtual Visualization For The Mechanical Trade." International Symposium on Automation and Robotics in Construction (ISARC), National Taiwan University, pp. 1131-1136.

2. Milgram, P. and Kishino, F. 1994. "A Taxonomy of Mixed Reality Visual Displays." IECE Trans. on Information and Systems (Special Issue on Networked Reality), Vol. E77-D, No. 12, pp. 1321-1329.

3. ARToolKit information website. http://www.hitl.washington.edu/research/share d_space/

4. Woo, M., Neider, J., Davis, T, and Shreiner, D. 1999. OpenGL Programming Guide, 3rd edition, OpenGL Architecture Review Board, Addison-Wesley Longman, Inc.

5. Shiau, Yan-Chyuan, Wang, Ming-Teh and Yang, Chung-Ham. 2001. "The Application of 3D and Interference Detection in Construction Engineering." Proceedings of the 18th
International Symposium on Automation and Robotics in Construction (ISARC). E.Bundy and J. Szlagowski, editors, pp. 95-100.

6. Tversky, Barbara, Lee, Paul and Mainwaring, Scott, 1999. "Why do speakers mix perspectives?" Spatial Cognition and Computation 1(4), pp. 399-412.

7. Levine, M., Jankovic, I.N. and Palij, M., 1982. "Principles of Spatial Problem Solving." Journal of Experimental Psychology General 111(2), pp. 157-175. 


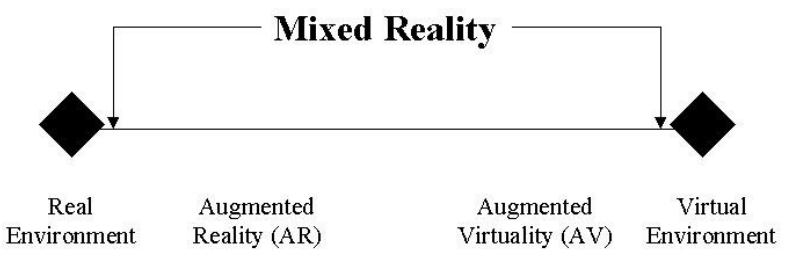

Figure 1. Mixed Reality encompasses all combinations of virtual and real.

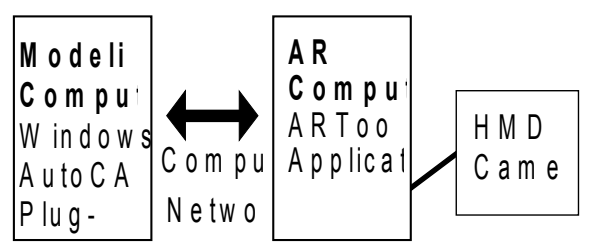

Figure 2. Components of the AR CAD prototype can operate on separate or a single computer.

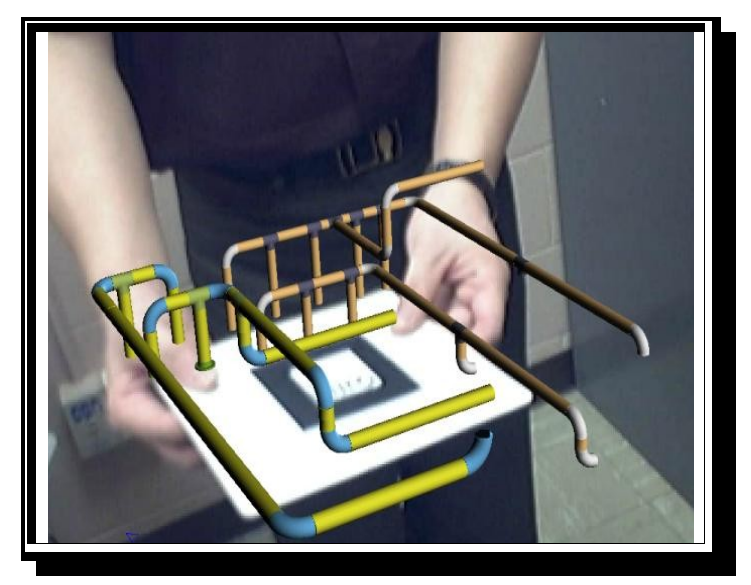

Figure 3. The real world scene is overlayed with a 3D virtual model.

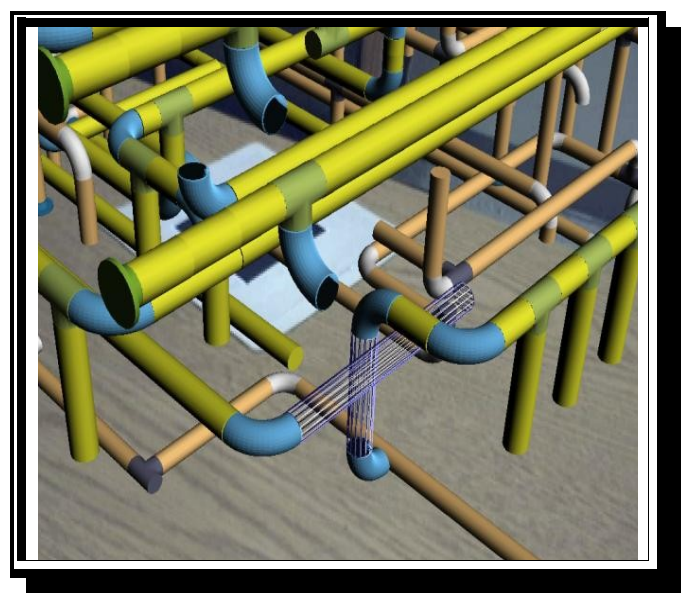

Figure 4. Wire frame representation identifies object interferences.

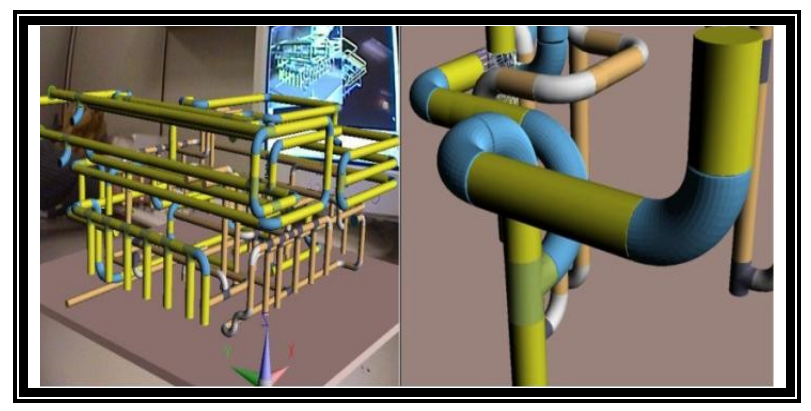

Figure 5. A zooming feature allows close up inspection.

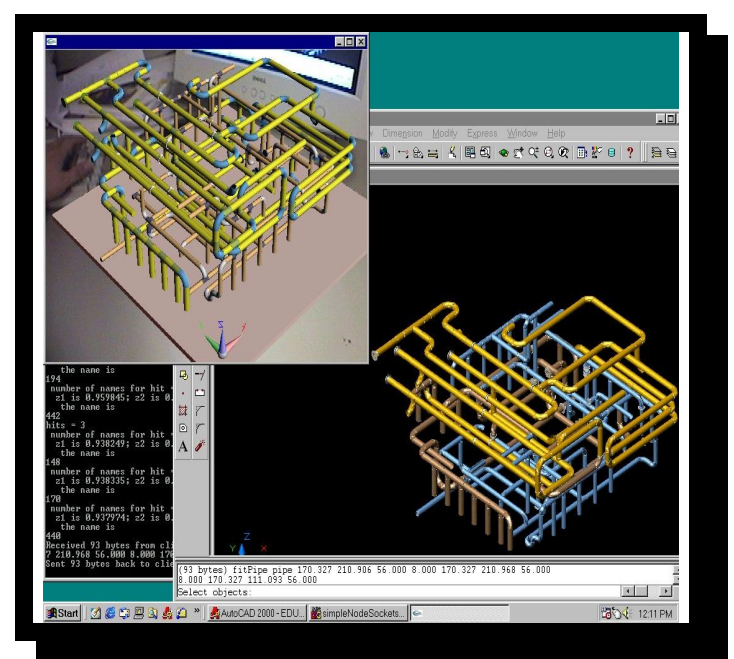

Figure 6. Views in AR and AutoCAD are not usually so aligned when switching scenes. 